\title{
Mobile customer relationship management: Factors affecting consumer mobile technology adoption within the hotel industry
}

\author{
Tina Y. T. Chan \\ This study was conducted under the supervision of Professor Statia Elliot, \\ School of Hospitality and Tourism Management, College of Management and Economics, \\ University of Guelph
}

\begin{abstract}
Mobile and smartphone technologies have been on the rise for a decade now, and their rapid adoption has compelled researchers to study the impact of this technological trend. While many of the studies focus on the general effects of mobile technology within the world of commerce, this paper aims to identify the factors that influence mobile customer relationship management by hotel brands. A multi-disciplinary review of research and literature identified several barriers and drivers of mobile technology adoption. The key drivers, immediacy, ubiquity and message personalization, were found to have a positive impact on mobile connections as they build trust and enhance satisfaction, thereby facilitating customer relationship management.
\end{abstract}

$\mathrm{W}$ ith the rising popularity of smartphones and mobile technology, businesses have been pushed to adapt their processes to this new generation of technology. While much research has been conducted to understand the effects of mobile technology within commerce, fewer studies have examined the impact from a consumer-relationship perspective within the hotel industry, despite the industry's dependence on their relationship with consumers. For many, it is as yet unclear whether mobile technology is facilitating or hindering these relationships. This paper will explore the emergence of mobile technology, in particular the rising trend of smartphone technology, and how it is impacting relationship marketing between hotel brands and their consumers.

\section{BACKGROUND}

\section{The Emergence of Mobile Technology}

As one of the fastest growing segments of mobile devices, the popularity of smartphones has increased significantly in recent years. Globally, approximately 302.6 million smartphones were purchased in 2010 [1]. Since the adoption of smartphone technology is relatively new, a limited amount of academic literature is available on mobile adoption within the hotel industry. Thus, this study is largely based upon a review of literature and research of general mobile technology adoption, with consideration of its applicability specifically within the hotel industry.

The main difference between smartphones and traditional mobile phones is the availability of data networks that allows users to do tasks that were not previously possible without physically using a computer [2, 3]. Smartphones, according to Raento, Oulasvirta, and Eagle [2], are programmable mobile phones that have sophisticated sensing capabilities, increased storage capacity and built-in networking functions. They also feature high-speed data connection, colour screens, cameras, local connectivity that enable web browsing, text and multimedia messaging, emailing, and social networking [2]. A study by the IDC Worldwide Digital Marketplace estimates that there are more than 450 million mobile web users worldwide and expects the figure to reach one billion users within four years [3]. Currently, leaders in the smartphone mobile device market are: Apple's iPhone, Research In Motion's BlackBerry, Google's Android, Nokia's Symbian and Microsoft's Windows Phone.

Since smartphone technology is still relatively novel, no standardized definition has been established for smartphone applications. For the purposes of this paper, smartphone applications are defined as third-party applications that can be downloaded and installed onto a smartphone. In 2010, a U.S. survey conducted by Luth Research and the Mobile Marketing Association found that more than 100,000 applications for iPhones have been downloaded over two billion times in just two years, with 40 percent of all adult consumers having downloaded at least one mobile application [3]. This popularity is indicative of consumers' acceptance of smartphone applications and their willingness to download such applications onto their personal mobile devices. According to Cortimiglia, Ghezzi and Renga [1], smartphone applications can be further categorized into three types based on their main function: 1) content-oriented 
applications that provide information, entertainment, communication, productivity and socialization, 2) marketingoriented applications that are mainly used for brand advertising or promotional purposes by companies and 3 ) service-oriented applications that allow users to perform specific self-serve tasks [1]. Another concept that has emerged with smartphones is mobile tagging. According to Canadi, Hopken and Fuchs [4], mobile tagging uses mobile device cameras to read the encoded data that is attached to physical objects and places as two-dimensional (2D) barcodes. These 2D barcodes or mobile codes, provide a standardized form of information for direct interaction (such as emails, contact information, websites) through hyperlinks, and are popular among retailers due to their cost efficiency and license-free usage [4].

With this technological evolution, several opportunities have developed within business contexts, especially in terms of mobile commerce (m-commerce) and mobile customer relationship management (mCRM). Derived from ecommerce, m-commerce is the application of wireless devices and data connection to conduct transactions where information, services or goods are transferred and exchanged [5]. Unlike e-commerce, m-commerce is very mobile and allows individuals to conduct such transactions virtually anywhere as long as they have access to a mobile device. Mobile customer relationship management, according to Sinisalo, Salo, Karjaluoto and Leppaniemi [6], is both oneway and interactive communication that is conducted via the mobile medium for the purpose of building and maintaining customer relationships between a business and its customers. With the amount of features that smartphones have to offer, there is an increase in opportunities now for businesses to communicate with their consumers, including smartphone applications and 2D codes. Like most innovative opportunities however, there are also challenges.

A challenge that arises with the advances in technology is technology itself. Technological advances are made on mobile hardware constantly, including improvements in battery capacity, central processing unit (CPU) power, display resolution, network connectivity as well as the variety of services offered on these mobile devices [7]. Since there are only limited amounts of CPU power and memory available on mobile platforms, it is more difficult to design and develop applications for the mobile market in comparison to desktop computer applications [7]. According to Fortier, Rossi, Fordillo and Challiol [8], some of the challenges with mobile context software include specific requirements, such as connectivity and location, that the software needs in order to process information. With the many different smartphone providers and their individual smartphone platforms, this makes it difficult for application developers to create an application that will function efficiently and smoothly on all types of mobile devices. By having these technical restrictions on mobile devices and wireless communication, the practical use of $\mathrm{m}$-commerce may be complicated [9]. This leads to the first question of this study:

Q1. Has the emergence of mobile technology made it more challenging for hotel brands to establish a relationship with their consumers?

\section{Hotel Industry Uses}

The popularity of mobile phones has allowed hotels to reach their customers and establish a relationship through a unique medium. In 2006, PhoCusWright and New York University conducted a study and found that fifty-three percent of hoteliers agreed that mobile technology would be important for their organizations in the next few years [10]. With the increase in mobile technology usage, many hotel brands have already begun utilizing mobile applications to stay in touch with their guests.

An early adopter, Starwood's W Hotels, launched a 'W To Go' short messaging service (SMS) text messaging application where guests are given the option of subscribing to receive reminder text messages during their stay at the hotels [10]. A spokesperson for $\mathrm{W}$ Hotels claims that the phone numbers collected through this service are used solely to enhance customer service and their experience at the hotel and not for promotional purposes [10].

Similarly, the economy-level hotel chain Days Inn, also uses text messaging as one of their marketing methods for targeted business guests [11]. Different Days Inn branches can send opt-in guests their daily hotel specials and restaurant coupons, allowing each hotel to customize to their own needs [11]. According to Days Inn's Vice President of Marketing, one of the main goals of the campaign is to create exposure for services that are not usually associated with an economy-level hotel [11]. Unlike W Hotels, Days Inn takes on a different approach with mobile technology and utilizes its text messaging application as a promotional medium.

As an example of a service-oriented application, the InterContinental Hotels Group has developed a mobile friendly version of their website so that guests can easily check the availability, book or cancel reservations directly from their mobile phones and other wireless devices [10]. These changes, according to the Vice President of IHG's Global E-commerce Department, were designed to provide their guests with convenience and ubiquity [10].

With some hotel brands, mobile marketing is limited to text messaging services while others have taken a step further to penetrate the smartphone market. For example, Small Luxury Hotels of the World (SLH), a collection of more than 500 luxury hotels across 70 countries, launched an application in 2010 for the iPhone [12]. This application allows guests to reserve hotels, share their experiences with others through Facebook, Twitter, and email as well as search for attractions nearby the hotel with personal recommendations from the staff [12]. In just over a year since the launch, this application has been downloaded 57,000 times and logged on by guests approximately 200,000 
times [12]. SLH's success shows the potential possibilities for hotels to utilize mobile technology as a medium to connect and communicate with their guests.

The different uses of mobile technology suggest that although mobile technology is still fairly new, hotels are beginning to adopt this technology. As a part of the service business, hotels value the relationships established with their guests and these relationships are maintained through customer relationship management, which leads to the second question of this study:

Q2. Will hotel brands that adopt the mobile technology trend be able to form a closer relationship with their consumers through applications and other smartphone technologies than hotel brands that have not adopted mobile technologies?

\section{RESEARCH METHODOLOGY}

A variety of literature was reviewed for this paper to attempt to capture findings relevant to the hotel industry. Journals within the fields of information technology and hospitality were primary sources. Additionally, academic articles, trade magazines and case studies from, marketing, service management and business process management fields were reviewed and findings were extrapolated to the hotels industry. These sources contribute to a greater understanding of mobile's influence within the as yet largely unexplored field of mobile hospitality.

\section{Factors Influencing Mobile Technology ADOPTION}

In order to examine consumers' willingness to adopt mobile technology, influencers for adoption are considered, and categorized into two main groups; those that 1) prevent and stall mobile technology adoption, or 2) push and increase consumers' utilization of mobile technology.

\section{Barriers}

According to Minghetti and Buhalis [13], having access to technology does not necessary mean that people will use it. Many people in fact have access but are unable to use technology effectively for a variety of reasons [13]. Of the various reasons, two main challenges that may prevent consumers from adopting mobile technology are technological challenges and perceived risks.

\section{Technological Challenges}

The world of technology is constantly developing and advancing. As mentioned by Verbelen et al. [7] earlier, application developers sometimes find it difficult to design applications for the mobile market because of the constant improvements that alter mobile devices. The same goes for consumers. With the constantly changing mobile platforms and interfaces, consumers may find it a challenge to keep up with the technological trends that are always developing, while some may even find it hard to accept available technologies. Van Dijk [14] presented a model to analyze the gap in the use of new technology between individuals and communities. In his model, he identifies an aspect known as "the skills access" which comprises of three types of skills needed for the adoption of new technology. These three types of skills are: instrumental digital skills that provide the capacity to work with hardware and software; information skills that allow the ability to search, select, and process information; and, strategic skills that permit the use of computer and network sources as the means for reaching goals and improving one's position in society [14]. Since mobile technology is still relatively new, in order for consumers to adopt this technology, they need to relearn these skills for the mobile market. This may become a problem for consumers, as there are constantly new mobile devices and applications developed. Consumers may find it troublesome to need to relearn these skills each time new mobile technology is released into the market.

In a study by Kim, Ma and Kim [15] of online hotel purchases, technological inclination was identified as a dimension that has significantly impacted consumers' satisfaction and purchase intentions, especially among consumers with no prior online purchase experiences. The authors found that the consumers' receptivity of new technological innovation and familiarity with e-commerce must be taken into account when evaluating their online purchase intentions. Although geared towards e-commerce rather than m-commerce, valuable insights from this study can be applied to m-commerce as well. When determining consumers' willingness to adopt mobile technology, their receptivity to mobile technology and familiarity with $\mathrm{m}$ commerce must be considered. If consumers are reluctant to accept this new form of technology and/or are not familiar with it, the possibility of them adopting mobile technology is likely to decrease.

\section{Risks}

Risks account for another major barrier to the adoption of mobile technology. In Forsythe and Shi's [16] study, they identified financial risk, psychological risk, physical risk, security risk, privacy risk and product performance risk as potential risks that consumers perceive when conducting transactions online. In fact, from a consumers' point of view, the largest barriers are related to data security and privacy threats [4]. Because of the similarities shared between ecommerce and m-commerce, these risks can also be considered relevant to transactions conducted on a mobile device as well.

Wang and Wang [17] conducted a study on the perceived risks that influence consumers when they book hotels through mobile technology. Three types of risks were identified in the study: 1) exposure of personal privacy information (such as financial and personal location information), 2) security risks due to unsafe wireless 
networks, and 3) unexpected product performance failures. Since mobile transactions are conducted through a wireless network that may or may not be secured in the eyes of the consumers. They may perceive that any information transferred through these network may be exposed to others, especially when transferred through an unsafe wireless network.

Despite the potential risks associated with mobile technology, empirical research found that perceived risks did not have a significant negative effect on the values involved with mobile hotel reservations [17]. Possible explanations were provided by Wang and Wang [17], who claimed that either increased exposures to online shopping experiences have provided consumers with the awareness of potential risks, or that they strongly confide with the payment procedures. In fact, in a previous study by Wong and Law [18], they found that one of the most successful factors for a online traveling website was providing secured payment methods. Although no significant negative impact was found between potential risks and the values with booking hotels online, potential risks still exist in the world of mobile technology. It is possible that consumers who are riskadverse may not want to adopt mobile technology because of the potential risks perceived, real or not.

\section{Drivers}

While there are barriers that exist in the mobile technological world, there are many drivers that help facilitate the adoption of these devices. Some of the main features of mobile technology include ubiquity/ reachability, information quality and immediacy, trust factors, and personalization/ compatibility. These characteristics are drivers of mobile technology, positively influencing consumers to adopt.

\section{Ubiquity/Reachability}

Ubiquity, is defined by the Collins English Dictionary [19], as the ability to be everywhere at once. It essentially fulfills the concept of being "anywhere" at "anytime". Mobile devices are considered personal devices. Unlike laptops and computers, they are often available at all times and are easily accessible by the user [20]. With mobile technology, users can gain access to desired information in spite of their place and physical location [9]. This provides users with the convenience to look up information as they travel without having to stop in between, interrupting their trip.

According to Balasubramanian, Peterson and Jarvenpaa [21], this ubiquitous characteristic of mobile technology is one of the main features of m-commerce. Because of mobile technology's ubiquitous nature, businesses are now given the opportunity to reach out to consumers regardless of their location or the time. In this sense, services and/or applications can also be made available to consumers wherever and whenever a need arises [9]. For example, Kannan, Chang and Whinston [20] suggest that instant feedback from consumers can now be obtained due to the fact that mobile technology is accessible to users at all times. With ubiquity, convenience is brought upon users as they can easily retrieve information anywhere at anytime. This feature can potentially increase consumers' mobile technology adoption levels.

\section{Informational Quality and Immediacy}

Another factor that may positively influence consumers' adoption to m-commerce and mobile technology is the information quality and the immediacy to retrieve such information. Wang and Wang [17] discovered that the ability to obtain information that is timely, detailed, accurate, reliable and selective has a significant and positive influence on perceived value when booking hotels through mobile hotel reservation systems. With mobile technology, users can easily acquire quality information through their devices in a timely fashion.

One of consumers' major uses of mobile technology within the hotels industry is to read hotel reviews. According to Pan, MacLaurin and Crotts [22], user-generated reviews on the Internet serve as a key source of information for travelers. A study by Ye, Law and $\mathrm{Gu}$ [23] found that positive online reviews can potentially increase the number of hotel bookings. For example, a 10 percent improvement in consumer ratings can increase sales by approximately 4.4 percent [23]. With the vast amount of travel review information on the web, mobile technology has enabled travelers to easily gain access to this information. Previous research also shows that immediacy in quality information has a vital effect on the number of Internet hotel sales. Kim et al. [15] conducted research on the determinants that affect Chinese hotel customers' online reservation intentions and their satisfaction towards these reservation systems. Through this study, they found that fulfilling information needs is significant for the satisfaction towards e-commerce, and if the information sought after is not available, it is likely the potential customer will search a different site instead [15]. Although this study focuses on e-commerce, the findings from the research could inform m-commerce due to their similarities. With the amount of noise presented in the media nowadays, consumers only attend to selective information and it is important for them to be able to get such information immediately. Since mobile technology enables quick transfers of quality information, this could be a factor that will encourage more consumers to adopt mobile technology.

\section{Trust}

Trust is a very important issue that may affect consumers' adoption of mobile technology. According to Yeh and $\mathrm{Li}$ [24], the level of satisfaction is the key to gain the trust of mobile users within the m-commerce world. In their study, satisfaction was defined as the "result of a process of post-purchase evaluation and comparison," and high satisfaction is achieved when the performance of a 
product is better than expected [24]. Findings show that trust towards mobile Internet vendors was significantly and directly affected by satisfaction and that consumers were more concerned with the brand image and familiarity of the mobile venders [24]. This indicates that trust within the mcommerce world can be built by enhancing the satisfaction level between the consumers' experience with m-commerce and the mobile vendors.

In Al-alak and Alnawas's [25] study, previous experience with advertising and the extensiveness of advertising were main determinants of trust towards permission-based marketing such as SMS marketing. It appears that consumers who have negative attitudes towards SMS marketing and those who were exposed to extensive advertising were less likely to participate in SMS marketing [25]. However, consumers who perceive permission-based advertising to be useful are more likely to trust the vendors and participate in such a program [25]. With mobile marketing, it is important to provide consumers with useful information in order to increase their satisfaction levels and hence the building of trust. It appears that consumers who trust the m-commerce world are more likely to adopt mobile technology.

\section{Personalization/Compatibility}

Another driver that could potentially aid the mobile technology adoption process is the personalization and compatibility aspect of mobile technology. Mobile devices are very accessible to the user and are often available at all times. Jiang [26] even suggests that mobile devices are more of lifestyle products rather than products of necessity and therefore, they are more compatible with users. This indicates that between all of the technological devices that exist in the market currently, mobile devices share the closest connection with users. This connection, in turn, allows highly personalized information to be shared between users and businesses.

For example, companies can use mobile technology as a marketing medium by sending SMS to consumers. This channel is quite valuable and helpful to businesses because it enables direct marketing activities [25]. In fact, Timpson and Troutman [27] discovered that advertisements sent via SMS are more likely to be viewed in comparison to advertisements sent through email. According to Frolick and Chen [28], because SMS is more personal, the expected response rate through mobile marketing will be more successful than through e-mail marketing. Lee and Mills [29] also state that personalization allows consumers' needs to be met more effectively and efficiently and thus, increases their satisfaction. Hence, the fact that personalization is present within mobile marketing mediums may cause consumers to adopt mobile technology.

\section{MOBILE CUSTOMER RELATIONSHIP MANAGEMENT AND IMPLICATIONS FOR HOTELS}

Sinisalo, Salo, Karjaluoto and Leppaniemi define mobile customer relationship management (mCRM) as [6]:

communication, either one-way or interaction, which is related to sales, marketing, and customer service activities conducted through the mobile medium for the purpose of building and maintaining customer relationships between a company and its customer(s).

With mCRM, the customer relationship is established and maintained through mobile technology. The drivers presented in this paper express the uniqueness of the mobile medium, which help strengthen the relationship between consumers and companies. Unlike other CRM channels, the ubiquitous nature of mobile technology allows the greatest access to consumers as they can be reached virtually anytime, anywhere [6]. Mobile technology also provides an instant transfer of information. As mentioned earlier, Starwood's W Hotels launched a 'W To Go' SMS text messaging application so guests can receive reminder text messages during their stay at the hotels [10]. This allows the formation of direct dialogues between the company and consumers [6], which will also be useful when immediate follow up and/or feedback are required to manage these relationships. The direct contact with the guests will allow personalization, thus making customer relationships easier to manage since mobile devices usually belong to one particular individual. For example, Days Inn's text messaging service markets towards guests that are already at the hotel [11]. Since it is expected that messages sent through mobile mediums will reach their intended audiences [6], Days Inn under this circumstance can tailor the content of the message to the needs of the guests. According to Nysveenm, Pedersen, Thorbjornsen and Berthon [30], the emotional relationships between a company and its customers can be strengthened if communication with loyal customers occurs on a personal and individual level. With the case of the iPhone application developed by Small Luxury Hotels of the World, guests are not only limited to reserving their stays using this application, they can also share their personal experiences with others onto their own social media sites [12]. Based on such findings, the answer to Q1 is no because the characteristics of mobile technology allow a better and more convenient method of customer relationship management that is tailored to each individual.

With mCRM, companies are now provided with a new channel to connect with their consumers. Sinisalo et al. [6] recommend companies to take advantage of this new channel but also to incorporate mCRM along with traditional CRM strategies as consumers can now choose the medium that they like, to interact with the companies. By offering more ways to communicate with consumers, customer 
relationships can be better maintained, thus, the answer to Q2 is supported.

\section{LIMITATIONS}

Though many studies have been presented here, there are limitations to be addressed in future research. First, the findings for this paper are not based on primarily research but rather, on empirical works of other researchers. Without properly conducting an experiment, it is difficult to conclude definitively what characteristics prompt consumers to adopt mobile technology. Future research should consider the implementation of a survey or other experiment to accurately determine the relative importance that these influencers have on mobile technology adoption. Second, some of the literature presented in this paper focuses on e-commerce as opposed to m-commerce. M-commerce is a relatively new concept, which continues to evolve as mobile technology increases in popularity. In particular, only minimal literature was found in regards to smartphone technology. It is expected that more literature on the topic of smartphone technology will appear within the next few years as smartphone use continues to grow. Third, as mentioned previously, not all of the literature found in this study is focused on the hotel industry because of the limited amount of public research conducted in this sector. Further investigations will be required to provide a deeper understanding of how mobile adoption affects the hotels industry.

\section{CONCLUSION}

Based on the research and literature reviewed for this study, it is clear that the rising trend of mobile and smartphone technologies have impacted the relationship between hotel brands and consumers. Evidence shows that the drivers of mobile technology can help fortify customer relationships, thus rejecting the first question posed that mobile technology has made it more challenging for hotel brands to connect with their consumers. This study also captured the influencers that affect consumers' adoption of mobile technology. It appears that the unique nature of mobile technology can increase consumer usage. As usage increases, businesses now have the opportunity to establish and strengthen relationships with their customers through this medium. This supports the answer to the second question posed: hotel bands that adopt mobile technology are able to form a much closer relationship with their consumers than hotel brands that have not adopted mobile technology.

This study contributes to the hospitality literature by identifying the influencers that can affect mobile technology adoption within the hotel industry. Trust is a very important issue in the m-commerce world. In order for consumers to foster a sense of trust, Al-alak and Alnawas [25] suggest providing consumers with useful information to increase their satisfaction levels. In Yeh and Li's [24] study, it was found that consumers' trust levels with m-commerce are heavily dependent on their satisfaction with their mcommerce experiences and the mobile vendors. In addition, because of the personal connections established between mobile devices and their users, highly personalized information can now also be communicated to users by businesses, making mobile technology a valuable tool for hotel brands to create bonds with their consumers.

\section{REFERENCES}

1. Cortimiglia, M.N., Ghezzi, A., \& Renga, F. (2011). Mobile applications and their delivery platforms. IT Professional Magazine, 13(5), 51-56.

2. Raento, M., Oulasvirta, A., \& Eagle, N. (2009). Smartphones: An emerging tool for social scientists. Socialogical Methods \& Reseach, 37(3), 426-454.

3. Goldman, S., M. (2010). Transformers. Journal of Consumer Marketing, 27(5), 469-473.

4. Canadi, M., Hopken, W., \& Fuchs, M. (2011). Virtualisation of customer cards with 2D codes. Information Technology \& Tourism 2011, 5, 215-227.

5. Kao, D.T. (2009). The impact of transaction trust on consumers' intentions to adopt M-commerce: A crosscultural investigation. CyberPsychology \& Behaviour, 12(2), 225-229.

6. Sinisalo, J., Salo, J., Karjaluoto, H., \& Leppaniemi, M. (2007). Mobile customer relationahip management: underlying issues and challenges. Business Process Management Journal, 13(6), 771-787.

7. Verbelen, T., Stevens, T., Simoens, P., De Turck, F., \& Dhoedt, B. (2011). Dynamic deployment and quality adaptation for mobile augmented reality applications. Journal of Systems and Software, 84(11), 1871-1882.

8. Fortier, A., Rossi, G., Gordillo, S.E., \& Challiol, C. (2010). Dealing with variability in context-aware mobile software. Journal of Systems and Software, 83(6), 915936.

9. Siau, K., Lim, E.-P., \& Shen, Z. (2001). Mobile commerce: Promises, challenges and research agenda. Journal of Database Management, 12(3), 4-13.

10. Derek, G. (2006). Going MOBILE. Hotels, 40(6), 42-42.

11. Dilworth, D. (2009). Days Inn brings mobile to its premier hotels. DM News, 31(8), 4-4.

12. Klie, L. (2011). Hotels go mobile for a better stay. Customer Relationship Management, 15, 15-15.

13. Minghetti, V., \& Buhalis, D. (2010). Digital divide in tourism. Journal of Travel Research, 49(3), 267-281.

14. van Dijk, J.A.G.M. (2006). Digital divide research, achievements and shortcomings. Poetics, 34(4-5), 221235.

15. Kim, W.G., Ma, X., \& Kim, D. J. (2006). Determinants of Chinese hotel customers' e-satisfaction and purchase intentions. Tourism Management, 27(5), 890-900. 
16. Forsythe, S.M., \& Shi, B. (2003). Consumer patronage and risk perceptions in Internet shopping. Journal of Business Research, 56(11), 867-875.

17. Wang, H.-Y., \& Wang, S.,-H. (2010). Predicting mobile hotel reservation adoption: Insight from a perceived value standpoint. International Journal of Hospitality Management, 29(2010), 598-608.

18. Wong, J., \& Law, R. (2005). Analysing the intention to purchase on hotel websites: a study of travellers to Hong Kong. International Journal of Hospitality Management, 24(2005), 311-329.

19. ubiquity. (n.d.). Collins English Dictionary - Complete \& Unabridged 10th Edition. Retrieved November 18, 2011, from Dictionary.com website: http://dictionary.reference.com/browse/ubiquity

20. Kannan, P., Chang, A., \& Whinston, A. (2001). Wireless commerce: Marketing issues and possibilites. Paper presented at the 34th Annual Hawaii International Conferences on System Sciences, Maui, Hawaii.

21. Balasubramanian, S., Peterson, R. A., \& Jarvenpaa, S. L. (2002). Exploring the implications of M-commerce for markets and marketing. Academy of Marketing Science, 30(4), 348-361.

22. Pan, B., MacLaurin, T., \& Crotts, J. C. (2007). Travel blogs and the implications for destination marketing. Journal of Travel Research, 46(1), 35-45.

23. Ye, Q., Law, R., \& Gu, B. (2009). The impact of online user reviews on hotel room sales. International Journal of Hospitality Management, (2009), 180-182.
24. Yeh, Y.S. \& Li, Y.,-M. (2009). Building trust in mcommerce: contributions from quality and satisfaction. Online Information Review, 33(6), 1066-1086.

25. Al-alak, B.A.M., \& Alnawas, I.A.M. (2010). Mobile marketing: Examining the impact of trust, privacy, concern and consumers' attitudes on intention to purchase. International Journal of Business Management, 5(3), 28-41.

26. Jiang, P. (2009). Consumer adoption of mobile internet services: An exploratory study. Journal of Promotion Management, 15, 418-454.

27. Timpson, S., \& Troutman, M. (2009). The importance of a layered privacy policy on all mobile internet sites and mobile marketing campaigns. International Journal of Mobile Marketing, 4(1), 57-61.

28. Frolick, M.N., \& Chen, L.D. (2004). Assessing mobile commerce opportunities. Information Systems Management, 21(2), 53-61.

29. Lee, J.K., \& Mills, J.E. (2010). Exploring tourist satisfaction with mobile experience technology. International Management Review, 6(1), 91-111.

30. Nysveen, H., Pedersen, P.E., Thorbjornsen, H., \& Berthon, P. (2005). Mobilizing the brand: The effects of mobile services on brand relationships and main channel use. Journal of Service Research, 7(3), 257-276. 